\title{
Successful Treatment of Adenoid Cystic Carcinoma with the Application of a High-Dose Stereotactic Body Radiotherapy Boost
}

\author{
Paweł Polanowski ${ }^{a} \quad$ Dorota Księżniak-Barana ${ }^{\mathrm{a}} \quad$ Aleksandra Grządziel $^{\mathrm{b}}$ \\ Agnieszka Pietruszka ${ }^{a}$ Ewa Chmielik ${ }^{c}$ Bolesław Pilecki ${ }^{a}$ \\ Natalia Amrogowicz ${ }^{a} \quad$ Karolina Gajdac Krzysztof Składowskia \\ a1st Radiation and Clinical Oncology Department, Maria Sklodowska-Curie National \\ Research Institute of Oncology, Gliwice, Poland; ' ${ }^{2}$ adiotherapy Planning Department, \\ Maria Sklodowska-Curie National Research Institute of Oncology, Gliwice, Poland; \\ Tumor Pathology Department, Maria Sklodowska-Curie National Research Institute of \\ Oncology, Gliwice, Poland
}

\section{Keywords}

Adenoid cystic carcinoma - Stereotactic radiotherapy $\cdot$ Radiosurgery boost $\cdot$ Nasopharynx tumour

\begin{abstract}
Background: Adenoid cystic carcinoma (ACC) should be treated with a surgical procedure. Unfortunately, in some cases, such procedures are impossible to perform. In that event, radiotherapy can be used as a form of radical treatment, although ACC is established as a radioand chemoresistant tumour. Therefore, unconventional fractionated radiotherapy needs to be considered. Case presentation: Here, we present a case study of a patient with an unresectable tumour of the choanae and nasopharynx treated with a stereotactic radiotherapy boost in combination with conventional radiotherapy. We achieved complete clinical regression after application of a $1 \times 18$ Gy boost followed by conventional radiotherapy at 50 Gy in 25 fractions. The early and late tolerance of this treatment were positive. During the 2-year follow-up, local and distant recurrence were not detected. Conclusions: This case represents an individualized, modern and safe approach to unresectable ACC. This is one of the first cases to show the use of a combination of stereotactic and conventional radiotherapy in radical, conservative cancer treatment.




\section{Background}

Adenoid cystic carcinoma (ACC) of the head and neck region accounts for less than $1 \%$ of all cancers of this region [1]. Despite the fact that the ACC occurs most often in the salivary glands, it can also arise in other head and neck locations [2]. ACC is a slow-growing malignant neoplasm that shows a tendency towards perineural spread and local recurrence. Although the risk of lymph node involvement does not exceed 10\%, ACC is characterized by a high risk of distant metastasis, especially to the lungs. Radical surgery with possible adjuvant radiotherapy is the standard treatment. The role of systemic treatment in this type of cancer is limited due to its chemoresistance. In cases where surgery is not possible (e.g., unresectable or inoperable tumours), radiation therapy can be used as a definitive treatment. The presented case report concerns an unresectable tumour of the choanae and nasopharynx, which was treated with a stereotactic radiotherapy boost followed by conventional radiotherapy.

\section{Case Presentation}

A 36-year-old male patient presented with periodic headaches for 6 years. A magnetic resonance imaging (MRI) scan of the head and neck revealed a contrast-enhanced lesion measuring $20 \times 17 \mathrm{~mm}$ located at the nasopharynx wall and the posterior choana on the right side. Additionally, a decline of the sphenoid bone on the right side was found on the contrastenhanced computed tomography (CT) scan. Histopathological examination showed lowgrade ACC. The patient underwent additional examinations (chest X-ray and abdominal ultrasound), which showed no distant metastases. Based on imaging tests and nasofibrescopy, the clinical stage was determined as cT2N0M0 [3]. The patient's case was presented at the Head and Neck Unit, where he was disqualified from surgery due to the inability to perform radical resection with appropriate operating margins. Afterwards, during the case conference, our team qualified the patient for radiation therapy. To intensify the radiation therapy and increase the chance of achieving local control, the patient was qualified for a stereotactic radiotherapy boost before the beginning of conventional treatment. An immobilization mask was fitted, and a CT scan without intravenous contrast in the supine position and MR scan with gadolinium intravenous contrast and $1 \mathrm{~mm}$ slice thickness for both methods were performed to accurately identify and contour the gross tumour volume $\left(\mathrm{GTV}_{\text {boost }}=6.18 \mathrm{~cm}^{3}\right)$. A 3-mm margin was added to the $\mathrm{GTV}_{\text {boost }}$ creating the planning target volume $\left(\mathrm{PTV}_{\text {boost }}=\right.$ $\left.14.95 \mathrm{~cm}^{3}\right)$. The clinical target volume $\left(\mathrm{CTV}_{50 \mathrm{~Gy}}=88.24 \mathrm{~cm}^{3}\right)$ included the nasopharynx, the posterior $1 / 3$ of the nasal cavity and nerve pathways (pharyngeal nerve, glossopharyngeal nerve and branches of cranial nerves IX and X) to the base of the skull. As before, the planning target volume $\left(\mathrm{PTV}_{50 \mathrm{~Gy}}=133.21 \mathrm{~cm}^{3}\right)$ was created by adding a $3-\mathrm{mm}$ margin to the $\mathrm{CTV}_{50 \mathrm{~Gy}}$. The radiotherapy planning process consisted of preparing two treatment plans: a stereotactic radiotherapy plan in the MultiPlan v.4.6.1 system (Accuray, Sunnyvale, CA, USA) and a conventional radiotherapy plan in the Eclipse system (Varian Medical System, Palo Alto, CA, USA). The next phase of planning included the fusion of both of the abovementioned plans in the Eclipse system. The stereotactic single-dose boost (18 Gy) was planned at a reference isodose of $90 \%$ with the application of Iris collimators $(15.00 \mathrm{~mm}, 20.00 \mathrm{~mm})$ and $1756 \mathrm{MV}$ flattening filter-free photon beams. The conventional plan was calculated for a fraction dose of $2 \mathrm{~Gy}$ and a total dose of $50 \mathrm{~Gy}$. The volumetric modulated arc therapy technique was used for two 6-MV flattening filter-free arcs with the use of a high-definition multi-leaf collimator. The dose distribution in the target volumes and organs at risk for the stereotactic and conventional radiotherapy are shown in the tables below (Table 1 and Table 2). We decided to accept

\section{Karger'}


Table 1. Doses and volume parameters of the stereotactic radiosurgery boost

\begin{tabular}{lccc}
\hline & Minimal dose, Gy & Mean dose, Gy & Maximal dose, Gy \\
\hline GTVboost & 18.90 & 19.43 & 20.00 \\
PTVboost & 17.32 & 19.15 & 20.00 \\
Spinal canal & 0.04 & 0.12 & 0.69 \\
Brainstem & 0.07 & 0.38 & 1.00 \\
Brain & 0.03 & 0.51 & 17.32 \\
Mandible & 0.04 & 0.77 & 7.59 \\
Parotid gland right & 0.05 & 0.94 & 5.22 \\
Parotid gland left & 0.17 & 0.77 & 3.99 \\
\hline
\end{tabular}

(Maximal dose in eyes, lenses, optic pathways and cochleae $<1.20 \mathrm{~Gy}$ ).

Table 2. Conventional radiotherapy - dose analysis

\begin{tabular}{lccc}
\hline & Minimal dose, Gy & Mean dose, Gy & Maximal dose, Gy \\
\hline CTV50 & 34.82 & 50.31 & 58.02 \\
PTV50 & 32.22 & 49.03 & 58.02 \\
Lens right & 0.88 & 1.09 & 1.38 \\
Lens left & 0.98 & 1.16 & 1.40 \\
Eye right & 0.75 & 1.50 & 5.95 \\
Eye left & 0.82 & 1.57 & 6.36 \\
Cochlea right & 13.84 & 29.06 & 49.47 \\
Cochlea left & 16.62 & 2.76 & 47.80 \\
Optic pathways & 1.57 & 18.89 & 5.89 \\
Brainstem & 1.45 & 3.81 & 53.72 \\
Brain & 0.17 & 6.14 & 44.12 \\
Mandible & 0.25 & 11.71 & 44.58 \\
Parotid gland right & 0.57 & 10.25 & 46.73 \\
Parotid gland left & 0.54 & 2.94 & 44.08 \\
Spinal canal & 0.03 & &
\end{tabular}

minimal doses of conventional radiotherapy in the CTV and PTV as a result of the air in the maxillary sinuses included in the target volumes (Fig. 1 and Fig. 2).

The treatment began with the stereotactic radiosurgery boost, realized on a Cyber Knife

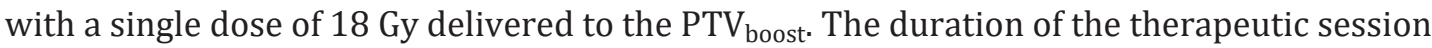
was $41 \mathrm{~min}$. A few hours after the boost, the patient reported a headache. Dexamethasone $2 \times 4 \mathrm{mg}$ i.v., paracetamol $1 \times 1$ g p.o., ketoprofen $1 \times 100 \mathrm{mg}$ i.v., ranitidine $1 \times 50 \mathrm{mg}$ i.v. and mannitol $1 \times 250 \mathrm{~mL}$ i.v. were administered. The headache subsided $1 \mathrm{~h}$ after administration of these drugs, but the pharmacological treatment was continued prophylactically for 5 days at the abovementioned doses. The steroid dose was reduced and changed to the oral route, and after 2 weeks from the beginning of treatment, steroids were discontinued. Conventional radiotherapy started 4 days after the stereotactic radiosurgery boost and was conducted at a total dose of $50 \mathrm{~Gy}$ in 25 fractions delivered to PTV $_{50 \mathrm{~Gy}}$ on a linear accelerator. The treatment was completed without any interruptions. Laboratory tests performed before treatment revealed increased levels of alanine transaminase (G1) and gamma-glutamyl transpeptidase (G1), most likely due to dietary error or alcohol consumption. The abdominal ultrasound showed no pathological changes, and L-ornithine L-aspartate $3 \times 100 \mathrm{mg}$ p.o. as a hepatoprotective treatment was administered, achieving normalization of those parameters. Grade 2 
Polanowski et al.: Successful Treatment of Adenoid Cystic Carcinoma with the Stereotactic Boost



Fig. 1. The dose distribution in the MultiPlan system. The orange isodose represents a prescription dose of $1 \times 18$ Gy (Cyber Knife).

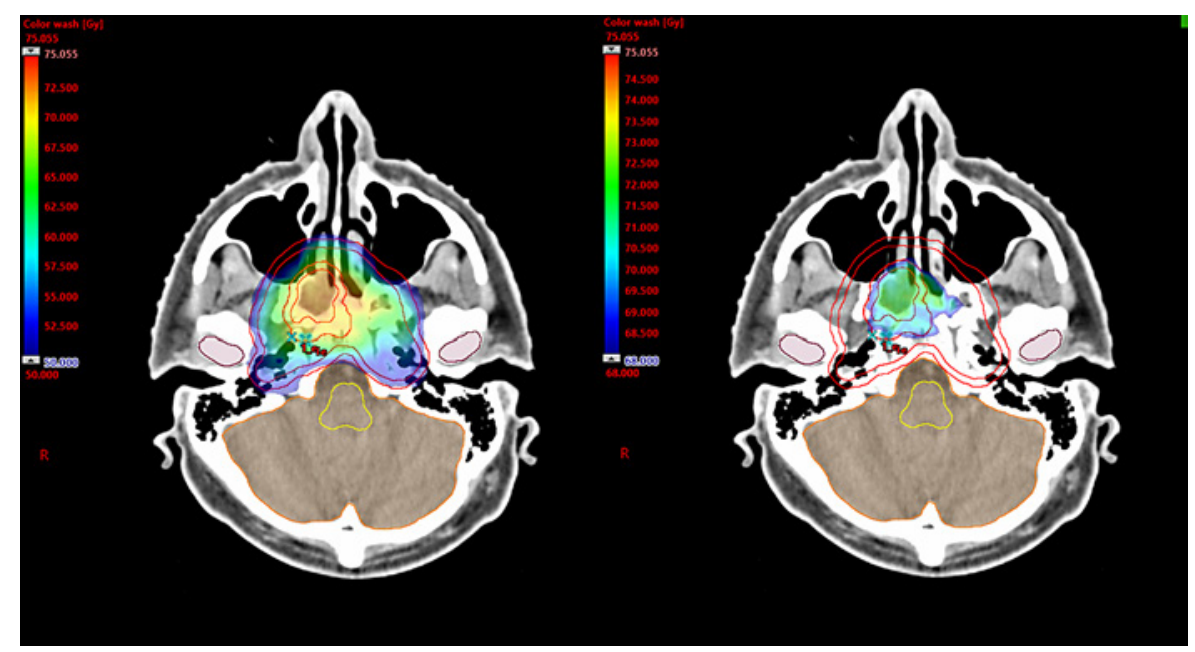

Fig. 2. The dose distribution in the Eclipse system (sum of the stereotactic radiosurgery boost and conventional radiotherapy). The dose was limited to $50 \mathrm{~Gy}$ (left) and $68 \mathrm{~Gy}$ (right).

mucositis of the nasopharynx and nasal cavity according to the Common Terminology Criteria for Adverse Events (CTCAE) occurred as acute side effects during conventional radiotherapy.

We performed two flexible nasal endoscopies (FNEs), the first 14 days after the start of treatment, and the second on the day before the last day of conventional radiotherapy. In both examinations, stagnation of tumour growth was observed with respect to the findings from nasal endoscopy performed before the treatment. In the first follow-up visit 2 months after the end of the treatment, a partial regression of the primary tumour in the FNE was observed; 


\section{Case Reports in Oncology}

Case Rep Oncol 2021;14:371-377

\begin{tabular}{l|l}
\hline DOI: $10.1159 / 000512069$ & ๑ 2021 The Author(s). Published by S. Karger AG, Basel
\end{tabular} www.karger.com/cro

Polanowski et al.: Successful Treatment of Adenoid Cystic Carcinoma with the Stereotactic Boost

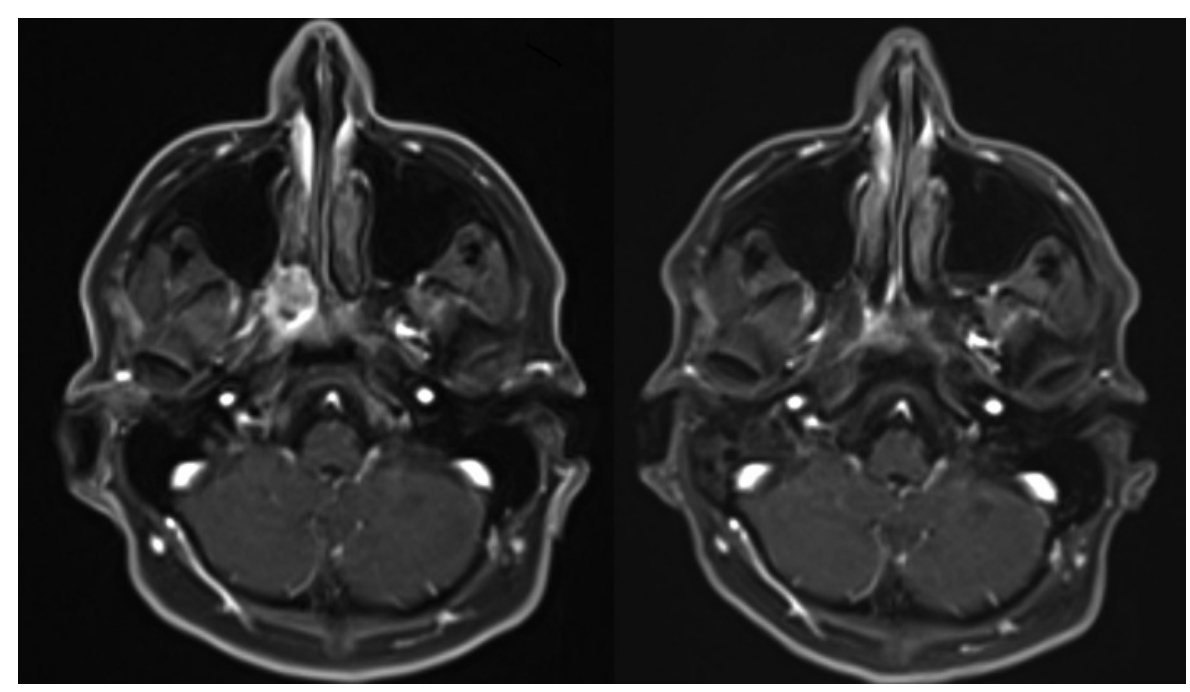

Fig. 3. Comparison of MR scans before (left) and 19 months after (right) the end of the treatment.

approximately $30 \%$ of the tumour remained. No evidence of acute side effects was found. Ten months after the end of the treatment, FNE demonstrated a normal nasopharynx and choanae region, indicating the complete remission of the part of the tumour that was visible in the previous FNEs.

More than 3 months after the end of treatment, the tumour dimensions on gadoliniumbased MRI were stable, simultaneously indicating evident response to radiotherapy due to the reduced diffusion restriction and the appearance of necrotic components in the tumour. Six months after the end of treatment, the tumour dimensions were similar to those on the previous MRI, $20 \times 14 \times 11 \mathrm{~mm}$. The radiologist suggested necrosis in the central part of the lesion as before and further reduction of diffusion restriction. Thirteen and 19 months after the end of the treatment, an abnormal $18 \times 15 \mathrm{~mm}$ area with low contrast enhancement was described on MRI, although there was no diffusion restriction on the MRI performed after 19 months, which can be interpreted as complete regression of the tumour with radiationinduced changes (Fig. 3). Chest X-ray and abdominal ultrasound revealed no distant metastases at the time. The patient underwent anterior tympanotomy and ventilatory drainage insertion 15 months after ending radiotherapy due to bilateral hearing impairment and chronic exudative, adhesive otitis media; this was the only late side effect of the treatment. Five months later, he reported significant improvement in hearing.

\section{Discussion}

ACC of the choanae and nasopharynx occurs casuistically. Despite the lack of data on large groups of patients, surgery with subsequent radiation therapy is a suggested method of treatment $[4,5]$. In the presented case, adequate surgical management was not possible due to the inability to perform an $\mathrm{R} 0$ resection. In such cases, radiotherapy remains the only option for radical treatment. Soprani et al. [6] described a case of ACC of the nasopharynx where after using conventional radiation therapy to a total dose of 70 Gy in 35 fractions, partial regression was achieved. Phan et al. [7] presented a case of a 71-year-old patient with nasopharyngeal ACC in grade T4N0 who was disqualified from surgery, and intensity modulated proton therapy was used to a total dose of 6,996 cGy in 33 fractions. However, 2 years 
after treatment, local recurrence occurred. American researchers have also used proton beam therapy for unresectable ACC of the nasopharynx [8]. In the reported group of 14 patients, the median dose was 73.8 Gy (relative biological effectiveness). Fifty percent of patients received concurrent chemotherapy. Three local, one regional and four distant failures were observed. The median time of local failure was 69 months. There was only one acute grade 3 toxicity and 3 patients developed grade 3 or higher late toxicity. Interesting conclusions were obtained by Schultz-Erter's study concerning patients with ACC infiltration of the skull base or orbital cavity [9]. In their study, at the 4-year follow-up, locoregional control was more beneficial after combined photon radiotherapy and a carbon ion boost than radiotherapy alone. The ACCEPT study also raised the problem of treating ACC in the head and neck region [10]. A group of 23 patients with different tumour locations (30\% paranasal sinus, $17 \%$ nasopharynx, $17 \%$ palate, $13 \%$ parotid gland, $9 \%$ submandibular gland, $13 \%$ others) showed quite good treatment results (only $22 \%$ local recurrences during the 4-year followup, 67\% 3-year disease-free survival) and acceptable tolerance (no grade 4 or 5 events) after administration of concurrent cetuximab and intensity modulated radiation therapy (conventional radiation therapy to a total dose of $54 \mathrm{~Gy}$ ) with an upfront carbon ion boost of $24 \mathrm{~Gy}$ in 8 fractions (relative biological effectiveness). Thirty-nine percent of patients were qualified to undergo surgical intervention as the primary treatment, but unfortunately, no radical surgery (R0) could be performed for any of the patients (R1 - 22\%, R2 - 78\%). These data show the difficulty of the surgical procedure in the treatment of ACC of the head and neck region. Typical ACC features such as resistance to radiation therapy and chemotherapy as well as difficulties in performing radical surgery should prompt the use of unconventional fractionation schemes in radiotherapy. In our case, the application of a stereotactic radiosurgery boost followed by conventionally fractionated radiation therapy was intended to increase the biologically effective dose in the tumour. The high gradient of dose fall-off outside the target, characteristic of stereotactic radiosurgery, enabled favourable dose distributions in the organs at risk.

\section{Conclusions}

This innovative, modern and safe method of treatment allowed us to achieve very good local control of the tumour. The combination of conventional radiotherapy with a stereotactic radiosurgery boost in the radical treatment of head and neck cancer requires further research to fully assess the efficacy and safety of this approach notably, in terms of late toxicity for instance necrosis, the risk of which is elevated after the application of stereotactic radiosurgery boost. The case described above suggests optimistic perspectives.

\section{Statement of Ethics}

The patient was informed about the unresectable character of the tumour. He gained information that radiosurgery boost may increase tumour response, especially in the radioresistance and chemoresistance aspect. The patient had the possibility to ask questions and he gained answers for all issues. Consent for radiotherapy treatment was obtained from the patient. Written informed consent was obtained from the patient for publication of this case report and any accompanying images. All authors read and approved the final manuscript. The datasets used and analysed during the current study are available from the corresponding author on reasonable request.

\section{Karger'k}




\section{Case Reports in Oncology}

\begin{tabular}{l|l}
\hline Case Rep Oncol 2021;14:371-377 \\
\hline DOI: 10.1159/000512069 & $\begin{array}{l}\odot \text { 2021 The Author(s). Published by S. Karger AG, Basel } \\
\text { www.karger.com/cro }\end{array}$ \\
\hline
\end{tabular}

Polanowski et al.: Successful Treatment of Adenoid Cystic Carcinoma with the Stereotactic Boost

\section{Conflict of Interest Statement}

The authors declare that they have no competing interests.

\section{Funding Sources}

Not applicable.

\section{Author Contributions}

Paweł Polanowski, Krzysztof Składowski, Natalia Amrogowicz: conception, realization of treatment, writing the manuscript; Dorota Księżniak-Baran, Agnieszka Pietruszka, Bolesław Pilecki: literature research; Aleksandra Grządziel: preparing of radiation therapy plan; Ewa Chmielik, Karolina Gajda: histopathological examination and immunohistochemical analysis.

\section{Acknowledgements}

We would like to thank Springer Nature Author Services for language service.

\section{References}

1 Batsakis JG, Luna MA, El-Naggar A. Histopathologic grading of salivary gland neoplasms: III. Adenoid cystic carcinomas. Ann Otol Rhinol Laryngol. 1990;99(12):1007-9.

2 Thompson LD, Penner C, Ho NJ, Foss RD, Miettinen M, Wieneke JA, et al. Sinonasal tract and nasopharyngeal adenoid cystic carcinoma: a clinicopathologic and immunophenotypicstudy of 86 cases. Head Neck Pathol. 2014;8(1):88-109.

3 Amin MB, Edge S, Greene F, Byrd DR, Brookland RK, Washington MK, editors, et al. . AJCC Cancer Staging Manual. New York, NY: Springer; 2017.

4 Liu TR, Yang AK, Guo X, Li QL, Song M, He JH, et al. Adenoid cystic carcinoma of the nasopharynx: 27-year experience. Laryngoscope. 2008 Nov;118(11):1981-8.

5 Wen SX, Tang PZ, Xu ZG, Qi YF, Li ZJ, Liu WS. [Therapeutic modalities of nasopharyngeal adenoid cystic carcinoma]. Zhonghua Er Bi Yan Hou Tou Jing Wai Ke Za Zhi. 2006 May;41(5):359-61.

6 Soprani F, Armaroli V, Venturini A, Emiliani E, Casolino D. A rare case of adenoid cystic carcinoma of the nasopharynx manifesting as Horner's syndrome: discussion and review of the literature. Acta Otorhinolaryngol Ital. 2007 Aug;27(4):216-9.

7 Phan J, Ng SP, Pollard C III2, Phan J. A Rare Case of Unresectable Adenoid Cystic Carcinoma of the Nasopharynx Treated with Intensity Modulated Proton Therapy. Cureus. 2017 Sep 15;9(9):e1688.

8 Gentile MS, Yip D, Liebsch NJ, Adams JA, Busse PM, Chan AW. Definitive proton beam therapy for adenoid cystic carcinoma of the nasopharynx involving the base of skull. Oral Oncol. 2017 Feb;65:38-44.

9 Schulz-Ertner D, Nikoghosyan A, Didinger B, Münter M, Jäkel O, Karger CP, et al. Therapy strategies for locally advanced adenoid cystic carcinomas using modern radiation therapy techniques. Cancer. 2005 Jul 15;104(2): 338-44.

10 Adeberg S, Akbaba S, Lang K, Held T, Verma V, Nikoghosyan A, et al. The Phase 1/2 ACCEPT Trial: Concurrent Cetuximab and Intensity Modulated Radiation Therapy with Carbon Ion Boost for Adenoid Cystic Carcinoma of the Head and Neck. International Journal of Radiation Oncology*Biology*Physics. 2020 Jan 1;106(1):16773. 